\title{
The Uptake of Sporopollenin Exine Capsules and Associated Bioavailability of Adsorbed Oestradiol in Selected Aquatic Invertebrates
}

\author{
Emma Chapman ${ }^{1}$ - Aimilia Meichanetzoglou ${ }^{2} \cdot$ Andrew N. Boa ${ }^{2} \cdot$ Hanne Hetjens ${ }^{3} \cdot$ Sonja Faetsch ${ }^{4}$. \\ Johnny Teuchies ${ }^{3}$. Sebastian Höss ${ }^{5}$. Dean Moore ${ }^{2} \cdot$ Lieven Bervoets $^{3} \cdot$ Paul Kay $^{6}$. Susanne Heise ${ }^{4}$. Paul Walker ${ }^{7}$. \\ Jeanette M. Rotchell ${ }^{1}$ (i)
}

Received: 9 March 2021 / Accepted: 22 August 2021 / Published online: 30 August 2021

(c) The Author(s) 2021

\begin{abstract}
Lycopodium clavatum sporopollenin exine capsules (SpECs) are known to both adsorb and absorb chemicals. The aim of the present work was to determine whether oestradiol (E2) is 'bioavailable' to bioindicator species, either pre-adsorbed to, or in the presence of, SpECs. SpEC uptake was confirmed for Daphnia magna and Dreissena bugensis. E2 levels varied among treatments for Caenorhabditis elegans though there was no relationship to SpEC load. E2 was not detected in D. bugensis tissues. Expression changes of general stress and E2-specific genes were measured. For C. elegans, NHR-14 expression suggested that SpECs modulate E2 impacts, but not general health responses. For D. magna, SpECs alone and with E2 changed Vtgl and general stress responses. For D. bugensis, SpECS were taken up but no E2 or change in gene expression was detected after exposure to E2 and/or SpECs. The present study is the first to investigate SpECs and bound chemical dynamics.
\end{abstract}

Keywords Sporopollenin $\cdot$ Oestrogen $\cdot$ Uptake $\cdot$ Bioavailability $\cdot$ Gene expression

Oestrogens enter the aquatic environment mainly via sewage treatment works (STWs) effluents. The most commonly found are the natural compounds, E1, E2 and E3 followed by synthetic EE2, none of which are significantly removed during STW clean-up processes (Racz and Goel 2010). Using the current UK population as an example, it has $\sim 38$ million

Jeanette M. Rotchell

J.Rotchell@hull.ac.uk

1 Department of Biological and Marine Sciences, University of Hull, Cottingham Rd, Hull HU6 7RX, UK

2 Department of Chemistry, University of Hull, Cottingham Road, Hull HU6 7RX, UK

3 Department of Biology, SPHERE, University of Antwerp, Groenenborgerlaan 171, 2020 Antwerp, Belgium

4 Hamburg University of Applied Sciences, Ulmenliet 20, 21033 Hamburg, Germany

5 Ecossa, Giselastr. 6, 82319 Starnberg, Germany

6 School of Geography/water@ leeds, University of Leeds, Leeds LS2 9JT, UK

7 SOCOTEC UK Ltd, Etwall House, Bretby Business Pk, Ashby Road, Burton on Trent DE15 0YZ, UK adults (not including the elderly), amounting to $167 \mathrm{~kg}$ of oestrogens arriving at STWs each year. A further $500 \mathrm{~kg}$ of consumed prescribed oestrogens each year can be added according to calculations by Stuer-Lauridsen and Kjolholt (2000). Such environmental oestrogens are deemed to pose a human and ecological risk at levels over 0.028 and $0.035 \mathrm{ng} \mathrm{L}^{-1}$ respectively, with links to elevated risk of cancer, cardiovascular disease and detrimental reproductive effects in humans (Adeel et al. 2017).

Pollens and spore grains are part of the plant reproductive system, with a double layered wall structure and exine layer, a resistant organic polymer, sporopollenin, of debated structure (Li et al. 2019; Mikhael et al. 2020). Sporopollenin can be extracted to provide empty shells, called sporopollenin exine capsules (SpECs). The surface of SpECs are penetrated by channels, giving a high surface area and absorption capacity (Thio et al. 2011; Rowley et al. 2011), thus potentially useful in removing chemicals at STWs. Here, we aimed to determine whether E2 is 'bioavailable' to a variety of bioindicator species from freshwater environments, once adsorbed to or in the presence of Lycopodium clavatum SpECs. The effects of E2 on aquatic invertebrates, quantified via LCMS-MS and gene expression, were investigated 
in E2-dosed water contrasting with E2-loaded SpECs to explore the potential application of SpECs as E2 adsorbents in wastewater treatment.

\section{Materials and Methods}

SpECs were extracted from spores of L. clavatum using $9 \mathrm{M}$ $\mathrm{HCl}$. SpECs ( $3 \mathrm{~g}$ ) were added to a solution of E2 in ethanol $(0.1 \mu \mathrm{g} / \mathrm{mL}, 30 \mathrm{~mL})$. The solvent was evaporated, then the solids resuspended in $10 \mathrm{~mL}$ ethanol, rinsing well the flask walls. Evaporation to dryness gave the E2-loaded SpECs (1 ng E2/mg). For exposure experiments, selection SpECs mass for a given volume gave, in theory, the concentrations of E2 if it all dissolved. Caenorhabditis elegans (Rhabditida, Rhabditidae) nematodes $(\mathrm{n}=\sim 400$ individuals, three replicates), Daphnia magna (Cladocera, Daphniidae) planktonic crustaceans ( $\mathrm{n}=25$ in three replicates) and Dreissena bugensis (Myida, Dreissenidae) quagga mussels ( $\mathrm{n}=6$ in three replicates) were exposed to the following treatment groups: (i) control; (ii) E2 $10 \mathrm{ng} \mathrm{L}^{-1}$; (iii) E2 $100 \mathrm{ng} \mathrm{L}^{-1}$; (iv) untreated SpECs; (v) E2-loaded SpECs. The masses of E2-loaded SpECs were chosen in each case to reflect the potential maximal concentration of E2 if it was fully desorbed (bioavailable) from the SpECs, and matched the E2 concentrations at low $\left(10 \mathrm{ng} \mathrm{L}^{-1}\right)$ and high dose $\left(100 \mathrm{ng} \mathrm{L}^{-1}\right)$ exposures. The same masses of untreated SpECs were also used. Exposure volumes used were $50 \mathrm{~mL}$ for $C$. elegans and D. magna and $500 \mathrm{~mL}$ for D. bugensis. Exposure duration, without feeding, was $24 \mathrm{~h}$ for C. elegans (at $20^{\circ} \mathrm{C}$ ), $48 \mathrm{~h}$ for D. magna $\left(20 \pm 1^{\circ} \mathrm{C}, 16: 8\right.$ light-dark cycle) and for $72 \mathrm{~h}$ for $D$. bugensis $\left(15 \pm 1^{\circ} \mathrm{C}, 16: 8\right.$ light-dark cycle). For $C$. elegans, all exposure solutions or suspensions were prepared in M9-medium ( $\mathrm{pH}=7.2)$. Exposure solutions were prepared with Millipore ultrapure water for D. magna and dechlorinated tap water for $D$. bugensis, $\mathrm{pH}=7.8$. On termination, one third of the D. magna and D. bugensis were fixed in $4 \%$ formaldehyde, one third (for all species) immediately frozen at $-80^{\circ} \mathrm{C}$ for chemical analysis, and one third (of all species) stored in RNALater at $-20^{\circ} \mathrm{C}$ for gene expression analysis. C. elegans was not analysed for SpEC body burdens because it is unable to ingest particles $>3 \mu \mathrm{m}$ (Fueser et al. 2019).

SpEC numbers in D. magna gut were counted and photographed under visible light and with a CY3 filter (orange) on an Olympus IX71 inverted microscope using CellSens Entry software (Olympus, UK). Statistical analysis (Kruskal-Wallis Test $(K W) \mathrm{n}=12-21$, followed by Dunn's comparisons with control group) was carried out in GraphPad InStat v3. D. bugensis, stored in $4 \%$ formaldehyde, were halved, washed, dehydrated, cleared, and embedded in paraffin wax. Sections $(10 \mu \mathrm{m})$ were dried overnight, cleared with Histoclear II and mounted with DPX medium. Three fields of view of each section were photographed and SpECs were counted. C. elegans $(\mathrm{n}=3$ pools of animals stored in ethanol of $600 \mu \mathrm{L}$ volume each, centrifuged and ethanol removal by pipetting/evaporation) and D. bugensis $(\mathrm{n}=5-6$ individuals from each exposure; $1.89 \pm 0.4 \mathrm{~g}$ wet weight), were pooled, homogenised and freeze-dried; $(0.2 \pm 0.06 \mathrm{~g}$ dry weight $)$. QuEChERS extractions were performed on the pooled $C$. elegans individuals and $D$. bugensis tissues. All samples were spiked with $100 \mathrm{ng} 17 \beta$-estradiol-2,3,4- ${ }^{13} \mathrm{C} 3$ internal standard (Sigma Aldrich, UK) in acetonitrile prior to extraction using QuEChERS (Phenomenex, UK). Samples were reconstituted with $300 \mu \mathrm{L} 100 \mu \mathrm{g} \mathrm{mL}^{-1}$ 4-(dimethylamino) benzoyl chloride (99\% purity) (Sigma Aldrich, UK) prepared in dry acetone with a 4-(dimethylamino)pyridine catalyst, and derivatised at $60^{\circ} \mathrm{C}$ for $1 \mathrm{~h}$, evaporated to dryness under nitrogen and reconstituted in $1 \mathrm{~mL}$ acetonitrile. These were filtered $(0.22 \mu \mathrm{m})$ and stored at $-20^{\circ} \mathrm{C}$. E2 analysis was conducted on a Shimadzu LCMS-8060 Triple Quadrupole Mass Spectrometer in positive ESI-MS/MS mode with quantification/qualifier MRMs at m/z 420-148/420-166 (DMABCE2) and 423-148/423-166 (DMABC $-{ }^{13} \mathrm{CE} 2$ ). The calibration curve was in triplicate from 0.01 to $10 \mathrm{ng} \mu \mathrm{L}^{-1}$ and calibration coefficients $\left(R^{2}\right)$ were 0.998 and 0.999 .

For gene expression analysis,total RNA extraction was performed on C. elegans and D. magna pooled from each experimental treatment. For $C$. elegans, this comprised $n=3$ pools of pelleted individuals $(\mathrm{n}=\sim 400$ per pool) from each exposure group. For D. magna, 14-28 individuals from each exposure group comprised one single pooled sample. D. bugensis, extraction was performed on $\sim 10 \mathrm{mg}$ of tissue from each experimental treatment. RNA was extracted with the High Pure RNA Tissue Kit (Roche, UK). C. elegans and D. magna had a proteinase $\mathrm{K}\left(20 \mu \mathrm{L} 600 \mathrm{U} \mathrm{mL}^{-1}\right.$, Thermo Scientific) and triton X-100 $(1 \mu \mathrm{L})$ incubation at $37^{\circ} \mathrm{C}$ for $15 \mathrm{~min}$, followed by snap freezing at $-80^{\circ} \mathrm{C}$ for $10 \mathrm{~min}$. After defrosting, the lysate was sheared through a 20-gauge needle five times before sonication. cDNA synthesis was performed using $170 \mathrm{ng}$ mussel RNA and $120 \mathrm{ng}$ of each $C$. elegans/D. magna RNA pool with the Precision Nanoscript2 Reverse Transcription Kit with random primers (PrimerDesign, UK).

qPCR assays were optimised for NHR-14 (nuclear hormone receptor 14), CAT (catalase), and reference genes pmp-3 (peroxisomal membrane protein) and $c d c-42$ (cell division control protein 42 homolog) for C. elegans. Vitellogenin 1 (Vtgl) and superoxide dismutase (SOD), and reference gene ubiquitin-conjugating enzyme $(U B C)$, were optimised for D. magna. For mussel, an ER product was obtained with primers 5'GATTATGTTTGTCCAGCTAC3' and 5'TTGTCAGGGTGGTATTTCTG3' based on Mytilus edulis MeER2 (GenBank AB257133.2). ER, CAT and reference genes $18 S, 28 S$ and $16 S$ were optimised for D. bugensis. qPCR reactions contained $10 \mu \mathrm{L}$ PrecisionPLUS $2 \times \mathrm{qPCR}$ MasterMix with SYBR Green for the ICycler (PrimerDesign, 
UK) $7 \mu \mathrm{L}$ molecular-grade water, $2 \mu \mathrm{L}$ primer mix and $1 \mu \mathrm{L}$ cDNA (from 120 ng RNA). Reactions were in duplicate on a CFX96 Real Time PCR Detection System (Bio-Rad, UK), with cycling conditions: $95^{\circ} \mathrm{C}$ for $2 \mathrm{~min}, 40$ cycles of $95^{\circ} \mathrm{C}$ for $10 \mathrm{~s}, 60^{\circ} \mathrm{C}$ for $1 \mathrm{~min}$ and $72^{\circ} \mathrm{C}$ for $1 \mathrm{~min}$. Reaction efficiencies were $90 \%-110 \%$ (Bustin et al. 2009). Relative mRNA expression levels were assessed using the $2^{-\Delta C T}$ version of the comparative $\mathrm{CT}$ method with the geometric mean of the reference genes (Livak and Schmittgen 2001) and statistically analysed in SPSS v24 ( $K W$ test, $\mathrm{n}=3-5)$.

\section{Results and Discussion}

SpECs were detected in the gut of D. magna from SpECsexposed treatments (Fig. 1b, c) (mean \pm SEM): Untreated SpECs $(19.05 \pm 2.86)$, Low E2 with SpECs $(14.33 \pm 2.96)$ and High E2 with SpECs $(16.42 \pm 3.93)$ (Fig. 1a) and were significantly higher than in the control group $(K W=91.45$, $\mathrm{df}=5, p<0.001$, Dunn's test $p<0.001$ ). The mean number of SpECs in non-SpECs exposed D. magna treatments was $<1$ and did not significantly differ from controls $(p>0.05)$ (Fig. 1a): Control $(0.57 \pm 0.18)$, Low E2 $(0.05 \pm 0.05)$ and High E2 (0). SpECs were also observed in the soft tissues of $D$. bugensis (Fig. 1d, f), but not in mussels exposed to control or E2 alone (Fig. 1e). A high degree of individual variation, in the randomly selected field of vision tissue sections, across the SpEC exposure groups, was detected (Fig. 1d).

The E2 analysis LOQ (peak signal to noise 10:1) was $0.02 \mathrm{ng} \mu \mathrm{L}^{-1}$ and LOD (peak signal to noise 3:1) was $0.01 \mathrm{ng} \mu \mathrm{L}^{-1}$. The recovery and derivatisation efficiency for labelled E2 varied widely between 0 and $49 \%$ due to the requirement of detecting derivatised E2 at low concentrations. For those samples at $0 \%$, or close to $0 \%$, the results were rejected and discarded. E2 levels in all $C$. elegans pooled samples for all exposure groups were

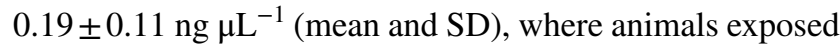

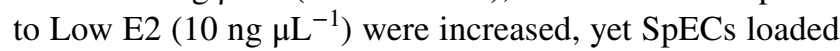
with High E2 (100 ng $\left.\mu \mathrm{L}^{-1}\right)$ were decreased, relative to controls (Fig. 2), though significance between groups is not quantifiable having used single pooled samples. E2 was not detected in any $D$. bugensis tissues.

mRNA expression of NHR-14 and CAT were investigated in single pools of $C$. elegans from: control, SpECs Low, SpECs High, Low E2, High E2, SpECs + Low E2, SpECs + High E2. Expression of NHR-14 in the treatments did not exceed that of the control group, with the exception of the SpECs + Low E2 treatment (Fig. 3a). CAT expression was lower in treatments compared to control with the
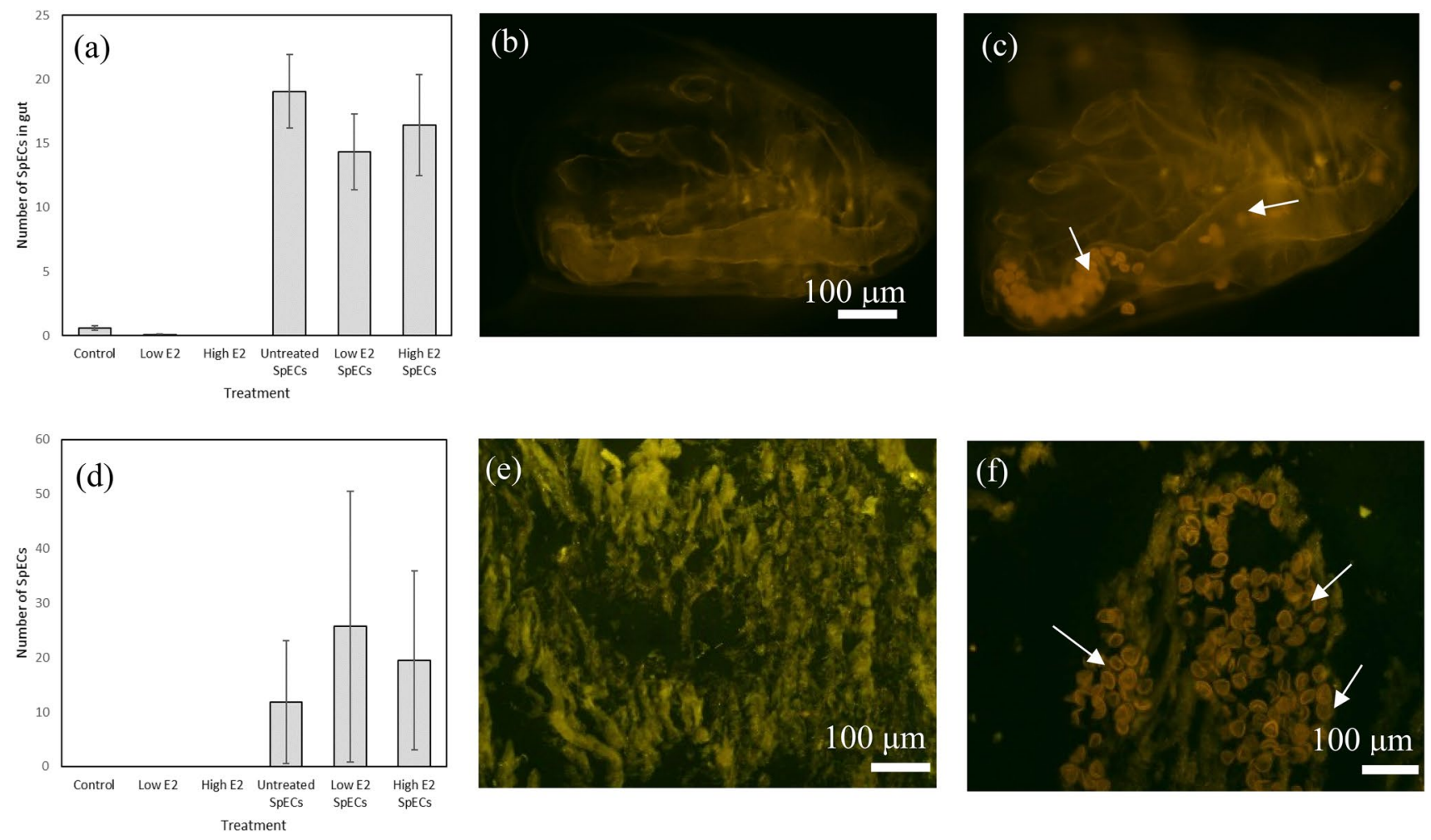

Fig. 1 Mean number of SpECs counted in a D. magna gut $(\mathrm{n}=12-21)$ and (d) randomly selected sections from D. bugensis whole body tissues $(n=6)$ from each treatment \pm SEM; and micro- graphs under CY3 fluorescence filter showing unstained whole $D$. magna and sections of D. bugensis from control $(\mathbf{b}, \mathbf{e})$ and SpECsexposed conditions (c, f). Arrows indicate SpECs 


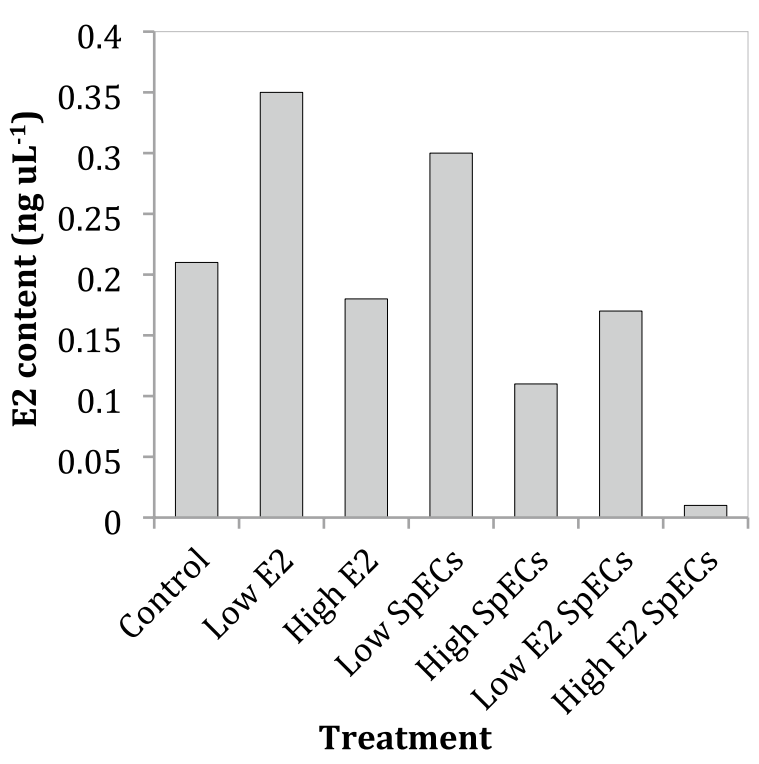

Fig. 2 Oestradiol content in pooled C. elegans samples $(\mathrm{n}=\sim 400$ per pool)

exception of the SpECs + High E2 treatment (Fig. 3b). Expression of $V \operatorname{tg} 1$ and $S O D$ were investigated in pooled samples of D. magna from the following conditions: Control, Untreated SpECs, Low E2, SpECs + Low E2, High E2, and SpECs + High E2 (Fig. 3c,d). Exposure to SpECs, either untreated (for $S O D$ ) or with E2 bound (Vtgl and $S O D)$, resulted in an increase in expression relative to the control or $\mathrm{E} 2$ alone groups. Expression of $E R$ and $C A T$ were investigated in D. bugensis tissues from: control, High E2, SpECs + High E2, and Untreated SpECs. No significant differences in expression were found between groups for either gene; $E R(K W=0.493, \mathrm{df}=3, p=0.92)$ and $C A T(K W=4.35$, $\mathrm{df}=3, p=0.226)($ Fig. 3e, f).

Histological analysis confirms the uptake of SpECs in $D$. magna and D. bugensis with significant numbers observed in the gut and other tissues, respectively for each species. Even if SpECs adsorb E2 to the extent that it is no longer bioavailable, their removal from wastewater before release into a receiving environment, such as a river beside a STW outfall source, may still be required due to the wider implications of potential uptake by organisms. For example, as exines are durable structures, they are resistant to degradation and digestion, so they will persist in the environment. The nutritional content of ingested grains is derived from the encapsulated cytoplasm, and this plus the digestibility of pollen grains varies among plant species and consumers (Roulston and Cane 2000). An exclusive diet of pollen was found to negatively affect development of zooplankton including D. longispina; whereby the presence of microorganisms is required to degrade them to an intermediate trophic level (Masclaux et al. 2011). As SpECs are empty capsules, they lack the nutritional component of intact grains and have been proposed as a dilution agent in artificial diets for arthropod larvae (Tainsh et al. 2020). The effects of dietary dilution by SpECs on invertebrates are subject to further investigation.

Having confirmed uptake of SpECs by two organisms, it is important to know whether any chemicals bound to the surface are bioavailable. Chemical analysis confirmed that the E2 levels in C. elegans samples varied between treatments, suggesting that E2 is present and at varying levels according to treatment group, though no relationship between SpEC and E2 loading values was observed. In contrast to $C$. elegans, no E2 was detected in any D. bugensis, tissues. C. elegans and D. bugensis are both invertebrates and the presence and functional role of vertebrate steroids is debated (Scott, 2012).

The variation of E2 tissue levels observed in the $C$. elegans across the treatment groups, resulted in an apparent increase in E2 in C. elegans exposed to low levels (but not high levels) of E2 alone, suggesting that such levels are internally regulated by these organisms. Furthermore, the exposure treatment using high levels of $\mathrm{E} 2$ bound to SpECs resulted in a decrease in tissue E2 levels, suggesting that the SpEC loading may have a protective effect and/or prevented $\mathrm{E} 2$ uptake, relative to $\mathrm{E} 2$ alone.

In contrast to the variable E2 levels detected in C. elegans, no E2 was detected in any of the D. bugensis tissues in any treatment group. For molluscan species, the evidence for modulated gene expression of the related oestradiol receptor (ER) and associated vitellogenin (VTG) egg yolk protein is debated. Some studies confirm presence and up-regulation of oestrogen-responsive genes, or increased levels VTG proteins, following controlled E2 exposure (Leonard et al. 2017) and others conclude that the receptor is non-constitutive in molluscs and other invertebrates (Thornton et al. 2003), having no functional, or at least, no reactive role in oestrogen chemical responses in such organisms (Scott, 2012). The levels of E2 in bivalves have been shown to vary throughout the year; the profile is synchronised with variations of oocyte diameter and gonad index (Osada et al. 2004). Subsequently, E2 is considered to exhibit a seasonal change associated with the reproductive cycle and to be involved in the regulation of several reproductive processes in bivalves such as vitellogenesis (Osada et al. 2004). On the other hand, possible explanations for the lack of induction in bivalves have been suggested (Thornton et al. 2003), including the rapid esterification of oestrogens to conjugates (Labadie et al. 2007), which may also explain the lack of oestrogens detected in the tissues. The role of oestrogens and their functional mechanism of action in bivalves are therefore far from clear, with the results obtained using $D$. bugensis seemingly further underlining this complexity. More exposures are required to elucidate the role that SpECs might play as a vector for adsorbed chemicals, using a chemical contaminant for which 
Fig. 3 mRNA expression (arbitrary units) of a NHR-14 and b CAT in C. elegans $(\mathrm{n}=1200), \mathbf{c}$ $V T G 1$ and $\mathbf{d} S O D$ in D. magna (n=14 or 28), and $\mathbf{e} E R$ and $\mathbf{f}$ CAT in D. bugensis $(\mathrm{n}=3-5$, mean $\pm \mathrm{SEM}$ ) (a)

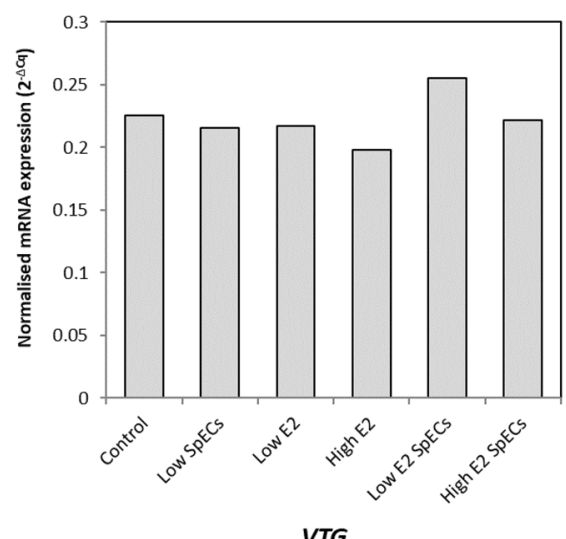

(c)

(e)

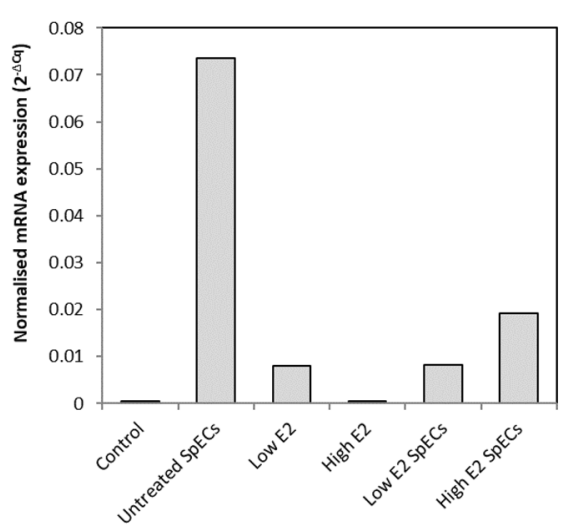

$E R$

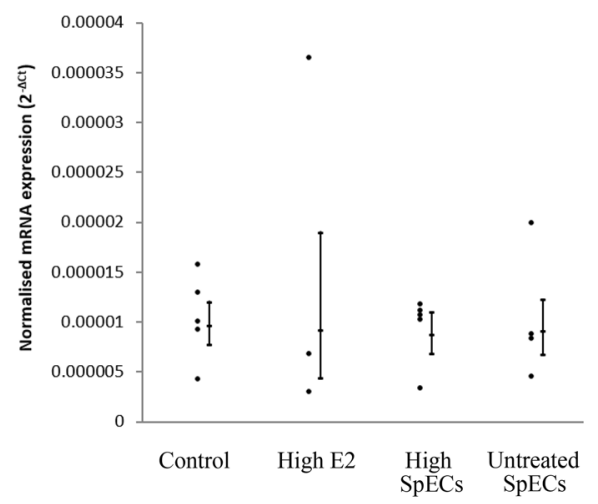

(b)

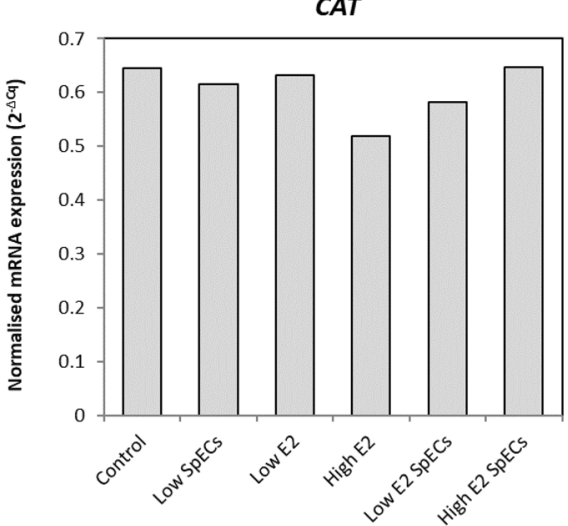

(d)

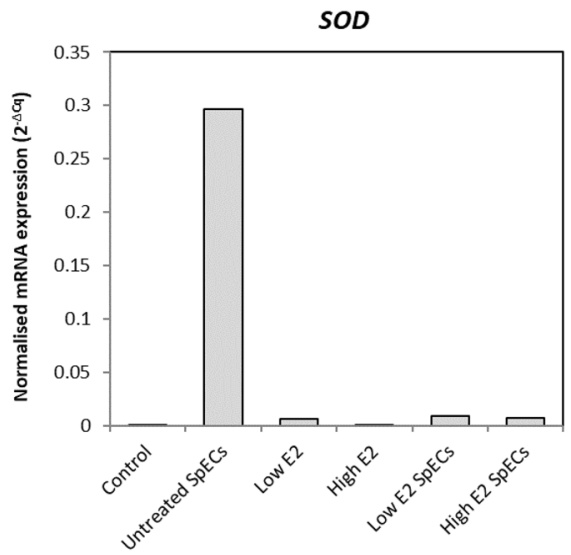

(f)

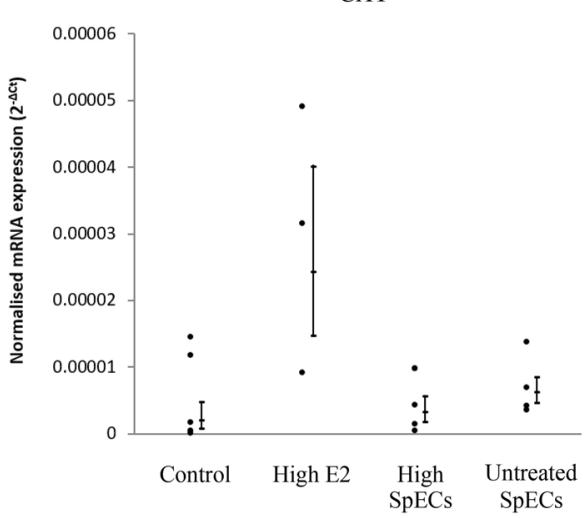

the uptake measurement is simple (avoiding recovery problems and derivatisation steps) and related to easily detected biological endpoints. This work represents a first attempt at monitoring the associated impacts of contaminants bound to SpECs and offers important insights into how to proceed in future studies.

The biological endpoints adopted herein relied on invertebrate species having an E2 specific and also general stress response to $\mathrm{SpEC} / \mathrm{E} 2$ exposure. Previous work conducted by Mimoto et al. (2007), using C. elegans in controlled laboratory exposures to oestrogenic chemicals, reported that expression of the NHR-14 can be up-regulated by oestrogens including E2. For these C. elegans, expression of NHR-14 in the SpECs + Low E2 treatment (Fig. 3) was the only exposure group to show E2 specific modulation of the gene. CAT mRNA expression, the general stress indicator, differed in the exposed treatment groups compared to the controls with the exception of the SpECs + High E2 treatment. These varying expressions suggest that E2 alone and E2 bound to SpECs do have differing biological impacts, but the pattern is unclear and further experiments, with more replicates is required.

Using daphnia for uptake and gene expression analysis is not a new approach (Heckmann et al. 2006), yet the 
mechanisms involved in the response to oestrogen exposure are not known, though the egg yolk protein vitellin has been shown to be induced by exposure (albeit at high levels of $1000 \mu \mathrm{g} \mathrm{L}^{-1}$ ) of EE2 (Clubbs and Brooks 2007). For both genes, exposure to SpECs, either untreated or with E2 bound, gave an increase in expression relative to the control or E2 alone groups (Fig. 3), highlighting the SpECs themselves, rather than E2, as the possible cause. The E2-specific endpoint Vtgl failed to indicate whether E2 was available or not in each exposure scenario. Daphnia Vtgl is also known to be elevated after thermal stress (Samanta et al. 2020), and inhibited by exposure to perfluoroethylcyclohexane sulfonate (Houde et al. 2016) or bisphenol A (Chen et al. 2021). In addition to Vtgl, Daphnia also possess another vitellogenin gene Vtg2 (Tokishita et al. 2006) but, similarly to these results presented, does not appear to respond to oestrogenic chemical activity (Hannas et al. 2011). More recently, the transcriptomic response of D. magna to E2 has been investigated revealing differentially-expressed genes with functions in immune responses, disease resistance, cancer-related functions and metabolism (Zheng et al. 2020). Such genes may provide alternative biomarkers for daphnia E2 exposure in future.

Gene expression studies are dependent on the extraction of high-quality template (Bustin et al. 2009), however this can be challenging when samples are small and/or contain structures resistant to extraction (Lienhard and Schäffer 2019). Daphnia contain a chitin carapace (Ebert 2005) interfering with extraction (Athanasio et al. 2016). C. elegans also has a protective collagen-like cuticle (Johnstone 1994). To combat these features, and the small body-size of both species, samples were pooled for extraction and RNA was quantified using a fluorescence-based method. RNA pooling has been used in microarray experiments where technical issues arise with low sample weights (Peng et al. 2003). The pooling of $C$. elegans and $D$. magna samples generates gene expression results that show trends which are not statistically significant results. Further replicates in future studies will compensate for the low body/tissue weights to increase RNA yield and avoid pooling.

To conclude, while the presence and implications of E2 in invertebrate organisms is unclear, the aim of this work was to determine whether it was bioavailable to the organisms, and once taken up, if it could subsequently cause a detrimental biological response. The uptake of SpECs has been confirmed by fluorescence microscopy for D. magna and D. bugensis. LCMSMS analysis also detected E2 variation among treatments for $C$. elegans, though no relationship to SpEC load was detected. A lack of E2 was detected in $D$. bugensis tissues suggests internal regulation. As an indicative biological response, the changes in gene expression of general stress biomarkers or specific to oestrogen chemicals were used. For C. elegans, the NHR-14 gene suggests that SpECs modulate E2 impacts, but not general health responses (measured by $C A T$ ) (Fig. 3). For daphnia, SpECs alone and combined with E2 suggest an effect on $V T G 1$ and stress response (as $S O D$ ) (Fig. 3). For D. bugensis no significant difference in expression was detected for $E R$ or $C A T$ in response to E2 and/or SpECs treatment (Fig. 3) and either alternative contaminants, or biological endpoints, must be pursued to better investigate the dynamics of SpECs and bound chemicals in these species.

Acknowledgements This work was funded by Interreg VB North Sea Region Programme to Sullied Sediments.

Open Access This article is licensed under a Creative Commons Attribution 4.0 International License, which permits use, sharing, adaptation, distribution and reproduction in any medium or format, as long as you give appropriate credit to the original author(s) and the source, provide a link to the Creative Commons licence, and indicate if changes were made. The images or other third party material in this article are included in the article's Creative Commons licence, unless indicated otherwise in a credit line to the material. If material is not included in the article's Creative Commons licence and your intended use is not permitted by statutory regulation or exceeds the permitted use, you will need to obtain permission directly from the copyright holder. To view a copy of this licence, visit http://creativecommons.org/licenses/by/4.0/.

\section{References}

Adeel M, Song X, Wang Y, Francis D, Yang Y (2017) Environmental impact of estrogens on human, animal and plant life: a critical review. Environ Int 99:107-119

Athanasio CG, Chipman JK, Viant MR, Mirbahai L (2016) Optimisation of DNA extraction from the crustacean Daphnia. PeerJ 4:e2004

Bustin SA, Benes V, Garson JA et al (2009) The MIQE guidelines: minimum information for publication of quantitative real-time PCR experiments. Clin Chem 55:611-622

Chen S, Li X, Li H et al (2021) Greater toxic potency of bisphenol AF than bisphenol A in growth, reproduction, and transcription of genes in Daphnia magna. Environ Sci Pollut Res Int. https://doi. org/10.1007/s11356-020-12153-5

Clubbs RL, Brooks BW (2007) Daphnia magna responses to a vertebrate estrogen receptor angonist and an antagonist: a multigenerational study. Ecotoxicol Environ Saf 67:385-398

Ebert D (2005) Ecology, epidemiology, and evolution of parasitism in daphnia [Internet]. National Library of Medicine (US), National Center for Biotechnology Information, Bethesda, MD. Available from: http://www.ncbi.nlm.nih.gov/entrez/query.fcgi?db=Books

Fueser H, Mueller M-T, Weiss L, Höss S, Traunspurger W (2019) Ingestion of microplastics by nematodes depends on feeding strategy and buccal cavity size. Environ Pollut 255:113227

Hannas BR, Wang YH, Thomson S, Kwon G, Li H, LeBlanc GA (2011) Regulation and dysregulation of vitellogenin mRNA accumulation in daphnids (Daphnia magna). Aquat Toxicol 101:351-357

Heckmann LH, Connon R, Hutchinson TH et al (2006) Expression of target and reference genes in Daphnia magna exposed to ibuprofen. BMC Genomics 7:175-182

Houde M, Douville M, Giraudo M et al (2016) Endocrine-disruption potential of perfluoroethyl cyclohexane sulfonate in chronically exposed Daphnia magna. Environ Pollut 218:950-956 
Johnstone IL (1994) The cuticle of the nematode Caenorhabditis elegans: a complex collagen structure. BioEssays 16:171-178

Labadie P, Peck M, Minier C, Hill EM (2007) Identification of the steroid fatty acid ester conjugates formed in vivo in Mytilus edulis as a result of exposure to estrogens. Steroids 72:41-49

Leonard JA, Cope WG, Hammer EJ, Barnhart MC, Bringolf RB (2017) Extending the toxicity-testing paradigm for freshwater mussels: assessing chronic reproductive effects of the synthetic estrogen $17 \alpha$-ethinylestradiol on the unionid mussel Elliptio complanate. Comp Biochem Physiol (C) 191:14-25

Li F-S, Phyo P, Jacobowitz J, Hong M, Weng J-K (2019) The molecular structure of plant sporopollenin. Nat Plants 5:41-46

Lienhard A, Schäffer S (2019) Extracting the invisible: obtaining high quality DNA is a challenging task in small arthropods. PeerJ 7:e6753

Livak KJ, Schmittgen TD (2001) Analysis of relative gene expression data using real-time quantitative PCR and the $2^{-\Delta \Delta C T}$ method. Methods 25(4):402-408

Masclaux H, Bec A, Kagami M et al (2011) Food quality of anemophilous plant pollen for zooplankton. Limnol Oceanogr 56:939-946

Mikhael A, Jurcic K, Schneider C et al (2020) Demystifying and unravelling the molecular structure of the biopolymer sporopollenin. Rapid Commun Mass Spectrom 34:e8740

Mimoto A, Fujii M, Usami M et al (2007) Identification of an estrogenic hormone receptor in Caenorhabditis elegans. Biochem Biophys Res Commun 364:883-888

Osada M, Tawarayama H, Mori K (2004) Estrogen synthesis in relation to gonadal development of Japanese scallop, Patinopectin yessoensis: gonadal profile and immunolocalization of $\mathrm{P} 450$ aromatase and estrogen. Comp Biochem Physiol (B) 139:123-128

Peng X, Wood CL, Blalock EM et al (2003) Statistical implications of pooling RNA samples for microarray experiments. BMC Bioinform 4:1-9

Racz L, Goel RK (2010) Fate and removal of estrogens in municipal wastewater. J Environ Monit 12:58-70
Roulston TH, Cane JH (2000) Pollen nutritional content and digestibility for animals. Plant Syst Evol 222:187-209

Rowley JR, Skvarla JJ, El-Ghazaly G (2011) Transfer of material through the microspore exine-from the loculus into the cytoplasm. Can J Bot 81:1070-1082

Samanta P, Im H, Shim T, Na J, Jung J (2020) Linking multiple biomarker responses in Daphnia magna under thermal stress. Environ Pollut 263:114432

Scott AP (2012) Do mollusks use vertebrate sex steroids as reproductive hormones? Part I: Critical appraisal of the evidence for the presence, biosynthesis and uptake of steroids. Steroids 77:1450-1468

Stuer-Lauridsen F, Kjolholt J (2000) Identification of selected hydrophobic organic contaminants in wastewater with semipermeable membrane devices (SPMDS). Water Res 34:3478-3482

Tainsh F, Woodmansey SR, Austin AJ, Bagnall TE, Gilbert JD (2020) Sporopollenin as a dilution agent in artificial diets for solitary bees. Apidologie. https://doi.org/10.1007/s13592-020-00801-1

Thio BJR, Clark KK, Keller AA (2011) Magnetic pollen grains as sorbents for facile removal of organic pollutants in aqueous media. $\mathrm{J}$ Hazard Mater 194:53-61

Thornton JW, Need E, Crews D (2003) Resurrecting the ancestral steroid receptor: ancient origin of estrogen signalling. Science 301:1714-1717

Tokishita SI, Kato Y, Kobayashi T, Nakamura S, Ohta T, Yamagata $\mathrm{H}$ (2006) Organization and repression by juvenile hormone of a vitellogenin gene cluster in the crustacean, Daphnia magna. Biochem Biophys Res Commun 345:362-370

Zheng Y, Yuan J, Gu Z, Yang G, Li T, Chen J (2020) Transcriptome alterations in female Daphnia (Daphnia magna) exposed to 17 $\beta$-estradiol. Environ Pollut 261:114208

Publisher's Note Springer Nature remains neutral with regard to jurisdictional claims in published maps and institutional affiliations. 